\title{
Subsecond Changes in Top-Down Control Exerted by Human Medial Frontal Cortex during Conflict and Action Selection: A Combined Transcranial Magnetic Stimulation- Electroencephalography Study
}

\author{
Paul C. J. Taylor, ${ }^{1,2}$ Anna C. Nobre, ${ }^{1,2}$ and Matthew F. S. Rushworth ${ }^{1,2}$ \\ ${ }^{1}$ Department of Experimental Psychology, University of Oxford, Oxford OX1 3UD, United Kingdom, and ${ }^{2}$ Centre for Functional Magnetic Resonance \\ Imaging of the Brain, Department of Clinical Neurology, University of Oxford, John Radcliffe Hospital, Oxford OX3 9DU, United Kingdom
}

Action selection requires choosing one of all the possible conflicting action plans that are available. There is currently a debate as to whether the dorsal medial frontal cortex (dMFC) merely detects or actively resolves response conflict. We used combined on-line transcranial magnetic stimulation and electroencephalographic recording (TMS-EEG) to test whether human dMFC plays a critical causal role in conflict resolution, and whether the mechanism for such a function is via interactions with primary motor cortex. In an Eriksen flanker task, subjects discriminated the direction of the centermost arrow in an array of five, responding with the left or right hand. The lateralized readiness potential (LRP), a measure of relative levels of activity in left and right motor cortices, was also recorded. Reaction times and error rates were higher on incongruent than congruent trials, and incongruent trials produced a positive LRP deflection reflecting initial partial activation of the incorrect response. On one-half of trials, repetitive TMS was applied to left dMFC starting $100 \mathrm{~ms}$ before visual stimulus onset and ending $100 \mathrm{~ms}$ afterward. TMS disrupted performance by selectively increasing error rates on contralateral (right hand) incongruent trials. TMS also only modulated the LRP on incongruent trials, causing an increased positive deflection (associated with preparation of the incorrect response) starting $180 \mathrm{~ms}$ after visual stimulus onset. TMS of a control site did not interfere with behavior or motor cortical activity. dMFC has a direct causal role in resolving conflict during action selection, and the mechanism involves the top-down modulation of primary motor cortical activity.

Key words: presupplementary motor area; TMS; ERP; task switching; attention; conflict

\section{Introduction}

Converging evidence suggests that human dorsal medial frontal cortex (dMFC), including presupplementary and supplementary motor areas (pre-SMA and SMA) and anterior cingulate cortex, may be specialized for processes involved in action selection, but its precise role and mechanism remain mysterious. Functional magnetic resonance imaging ( $\mathrm{fMRI}$ ) studies have implicated $\mathrm{dMFC}$ areas such as pre-SMA in higher-order motor selection during free selection of task sets and switching between action rules (Brass and von Cramon, 2002; Bunge et al., 2003; Forstmann et al., 2005; Crone et al., 2006a,c; Dosenbach et al., 2006). Action selection in such tasks requires choosing one of all possible and conflicting action plans available. Although dMFC activity increases when there is conflict between actions, it is not clear whether it is critical for resolution of conflict, or only for moni-

Received Feb. 19, 2007; revised July 17, 2007; accepted July 24, 2007.

This work was supported by the Medical Research Council, the Royal Society (M.F.S.R.), and the Biotechnology and Biological Sciences Research Council (A.C.N.). We are grateful to Mariagrazia Capizzi for technical assistance with data collection.

Correspondence should be addressed to Paul C. J. Taylor, The Henry Wellcome Building, School of Psychology, Birkbeck College, Torrington Square, London WC1E 7HX, UK. E-mail: pc.taylor@psychology.bbk.ac.uk. DOI:10.1523/JNEUROSCI.2877-07.2007

Copyright $\odot 2007$ Society for Neuroscience 0270-6474/07/2711343-11\$15.00/0 toring conflict. According to some accounts (Posner and Petersen, 1990), dMFC exerts top-down down control over sensorimotor systems to influence which action is selected. It has, however, proved difficult to provide direct evidence of such topdown control; this requires demonstrating that $\mathrm{dMFC}$ manipulations alter sensorimotor area activity and that the impact is greatest in a particular cognitive state, the state of action conflict.

Activity in a region can be inferred to have a causal role in a task from microstimulation and TMS studies in macaques and humans, respectively. Whereas dMFC microstimulation tends to facilitate performance, particularly on conflict trials (Stuphorn and Schall, 2006; Isoda and Hikosaka, 2007), TMS might be expected to disrupt performance. The activity induced by the TMS pulse is unlikely to correspond to a meaningful pattern and functions as interfering noise (Walsh and Rushworth, 1999; Walsh and Cowey, 2000). As Isoda and Hikosaka (2007) explain, it is difficult to train monkeys on the same complex tasks that are used with human subjects. Moreover, it remains unclear from microstimulation studies whether dMFC exerts its effect on conflict trials via top-down modulation of other areas.

Here, we extend the interference approach into the human domain and test whether TMS can reveal an active causal role for $\mathrm{dMFC}$ in a flanker task used in many human neuroimaging ex- 
periments to manipulate conflict (Eriksen and Eriksen, 1974; Botvinick et al., 2004). Second, this study also investigates the mechanism by which dMFC operates by deriving the lateralized readiness potential (LRP), an EEG measure of relative levels of activity in lateral motor cortex in each hemisphere when subjects choose between left- and right-hand responses (see Materials and Methods, Experiment 1: dMFC TMS, Analysis) (see Fig. 3) (Coles, 1989). Normally, response selection is accompanied by a negative LRP, reflecting greater activation contralateral to the moved hand. When response conflict is high, however, a positive LRP deflection reflects activation contralateral to the undesired response. We reasoned that, if $\mathrm{dMFC}$ exerts its influence in conflict tasks via top-down control over lateral motor regions, then dMFC TMS should affect the LRP. The experiment therefore tests whether it is possible to demonstrate that two regions of human cortex show changes in functional connectivity that occur on a subsecond scale and that are dependent on cognitive state.

\section{Materials and Methods}

\section{Experiment 1: dMFC TMS}

Participants. Sixteen right-handed subjects (11 females; mean age, 25; range, 20-36) participated in experiment 1. All subjects gave informed consent before participating in the study, which was approved by the Central Oxfordshire Research Ethics Committee (OxRec No. 05-Q1606-96).

Task design. In an Eriksen flanker task (Eriksen and Eriksen, 1974), subjects discriminated the direction of the centermost arrow (pointing to the left or right), presented in an array with four irrelevant distractor arrows, and responded as quickly as possible with the index finger of the corresponding left or right hand (Fig. 1). The central arrow pointed in either the same [congruent (e.g., " $<<<<<$ ")] or the opposite direction [incongruent (e.g., " $>><>>$ ")] to the distractors. This has been repeatedly shown to be an effective manipulation of conflict: behavioral performance, as indexed by slower reaction times (RTs) and more frequent errors, is worse on incongruent trials, reflecting the additional processing required to select the appropriate response in the context of the competing response elicited by the distracting flankers (Botvinick et al., 2004).

Trials were divided into equal numbers of congruent and incongruent distractors, with TMS and without TMS, and in which the correct response was with the left or the right hand. There were no more than two consecutive TMS trials, but otherwise trial order was pseudorandomly determined (identical for all subjects). On each trial, the visual stimulus array of five arrows was flashed for $100 \mathrm{~ms}$, preceded and followed by fixation crosses lasting $1000 \mathrm{~ms}$ each. A blank screen filled the intertrial interval, which was long $(7900-8900 \mathrm{~ms})$ to satisfy safety considerations regarding the minimum intertrain and rate of TMS application (Jahanshahi et al., 1997; Wassermann, 1998). Subjects were instructed to fixate and not to blink while stimuli were on the screen, and were trained on the task for either $5 \mathrm{~min}$ or until responses were accurate and consistent, whichever was the longest. Each arrow was $1^{\circ}$ of visual angle wide, $1.7^{\circ}$ tall, and the array was $5.3^{\circ}$ wide. Subjects sat in a darkened booth $100 \mathrm{~cm}$ from the screen and wore earplugs (signal-to-noise ratio, 28). All stimuli were white and presented on a black background. A total of 400 trials was presented in four blocks of 100 .

EEG recording. EEG was recorded continuously (NuAmps digital amplifiers; Neuroscan, El Paso, TX) (low-pass filter direct current, $300 \mathrm{~Hz}$; $1000 \mathrm{~Hz}$ sampling rate) from 30 scalp sites using $\mathrm{Ag} / \mathrm{AgCl}$ electrodes mounted on an elastic cap (Easy Caps, Herrsching-Breithrunn, Germany) according to the 10-20 international system. The montage included two midline sites (CZ, PZ), 14 sites over each hemisphere (F3/F4, FC3/FC4, FC5/FC6, C1/C2, C3/C4, C5/C6, CP1/CP2, CP3/CP4, CP5/ CP6, P3/P4, P5/P6, PO3/PO4, PO7/PO8, O1/O2), left and right mastoids, and two HEOG and two VEOG electrodes to monitor the electrooculogram (EOG) bipolarly. Additional electrodes were used as ground (FPZ-FZ) and reference (nose).

TMS. On one-half of trials, repetitive TMS was applied to left dMFC

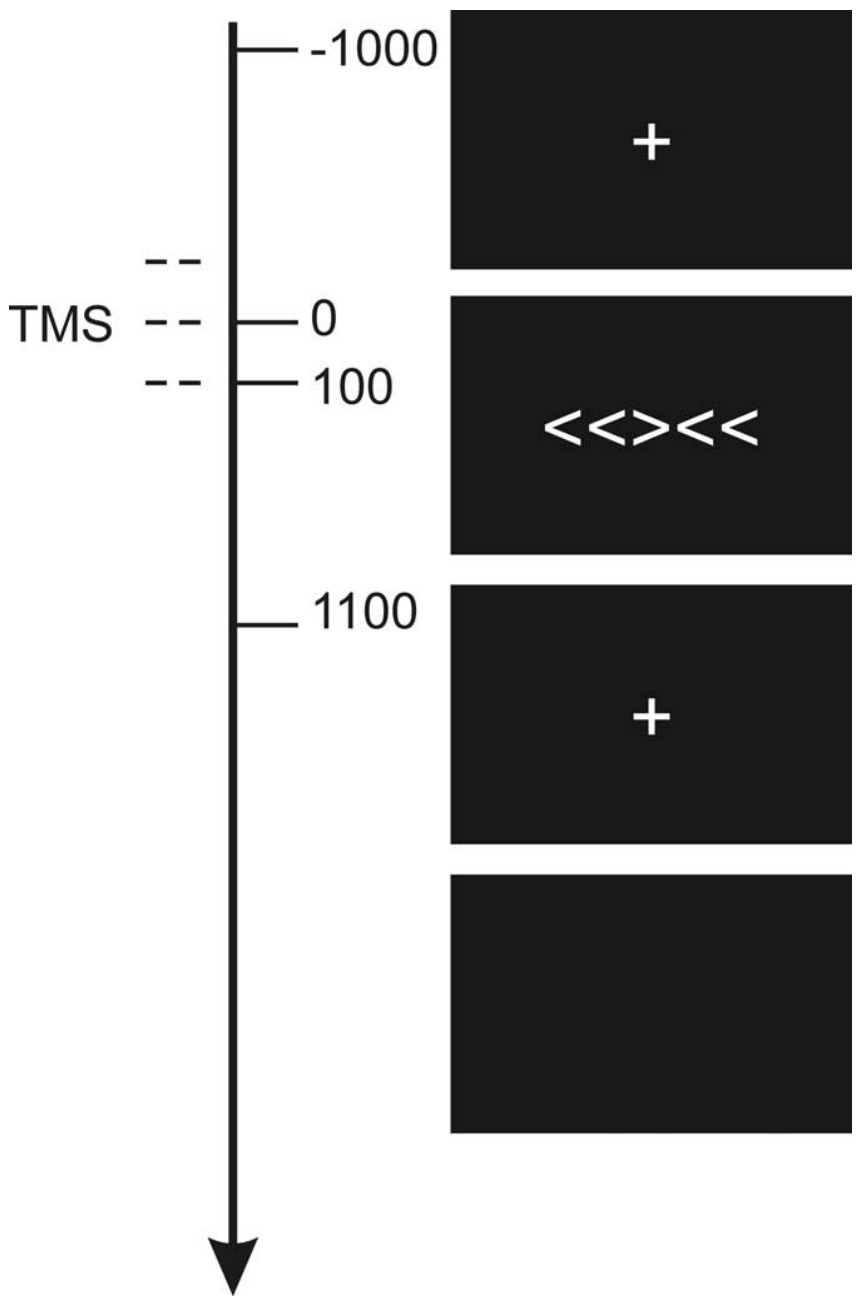

Figure 1. Task. Subjects discriminated the direction in which the central arrow was pointing (left or right) and responded with the corresponding hand (left or right, as appropriate). On TMS trials, stimulation was applied at $-100,0$, and $+100 \mathrm{~ms}$ relative to onset of the arrow array.

starting $100 \mathrm{~ms}$ before visual stimulus onset and ending $100 \mathrm{~ms}$ afterward (three pulses at $10 \mathrm{~Hz}$ and $110 \%$ motor threshold) using a figure 8 flat coil with an internal diameter of $7 \mathrm{~cm}$ (Magstim Rapid Machine, Whitland, Wales, UK). The dMFC TMS site was $5 \mathrm{~mm}$ lateral, to the left, of electrode position FCZ. Coil positioning was confirmed after the recording session by using the Brainsight frameless stereotaxy system (Brainsight, Magstim) for 5 subjects (Fig. 2a). All lay in dMFC and in close proximity of one another in Montreal Neurological Institute (MNI) space close to the area usually described as the presupplementary motor area (mean, $x=-5, y=7, z=73$ ). TMS parameters were all within the established safety guidelines and were approved by the Central Oxfordshire Research Ethics Committee (OxRec No. 05-Q1606-96).

Analysis. The analysis compared TMS and no-TMS trials, but care was taken to focus only on those TMS and no-TMS trials that had themselves been preceded by a no-TMS trial. Sequential trial-to-trial changes in levels of conflict are known to occur in tasks such as the Eriksen flanker task (Gratton et al., 1988). Conflict, as indexed by RT and error rate, is reduced on conflict trials that are preceded by a previous conflict trial in comparison with those conflict trials that are preceded by nonconflict trials. It is possible that TMS on the preceding trial could have augmented such effects but the potential confound was avoided by the exclusion of trials that had been preceded by a previous TMS trial, and any TMS effects were thus only attributable to the delivery of TMS on the current trial.

Event-related potentials (ERPs) were constructed off-line, using epochs starting $201 \mathrm{~ms}$ before visual stimulus onset and ending $400 \mathrm{~ms}$ 


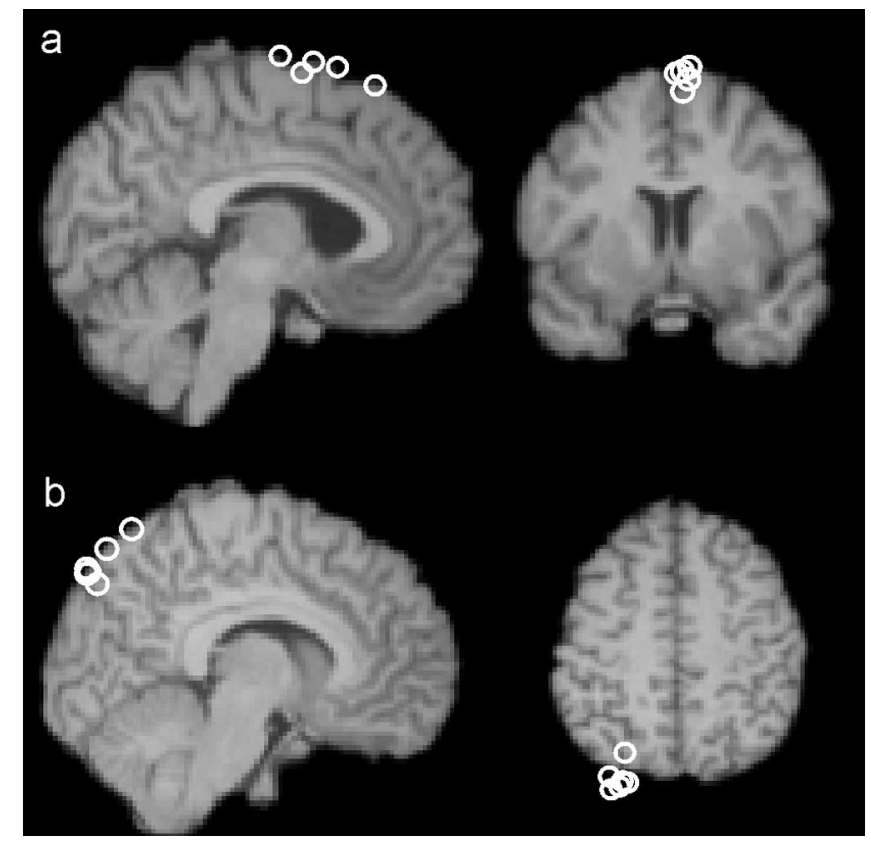

Figure 2. $\quad \boldsymbol{a}$, Left dMFC TMS site. The circles represent the MNI coordinates at which TMS was applied over left dMFC in a subset of the subjects from experiment 1 (mean, $x=-5, y=7$, $z=73$ ). The circles are superimposed over the brain of an example subject that had also been registered into $\mathrm{MNI}$ space. The site is just left of the midline and over tissue normally assigned to the pre-SMA. $\boldsymbol{b}$, The circles represent the MNI coordinates at which TMS was applied over the control site in a subset of the subjects from experiment 2 (mean, $x=-6, y=-81, z=52$ ).

afterward. Epochs were excluded if showing recording artifacts, if saccades or blinks occurred in the EOG, or if the subjects made errors [i.e., responses with the incorrect hand, early responses $(<200 \mathrm{~ms}$ after visual stimulus onset), or late responses $(>1200 \mathrm{~ms})]$. A minimum criterion of 20 trials per condition per subject was set to ensure high-quality ERP data (mean number of trials per subject per condition, 45). Data from two subjects were rejected because not enough trials survived artifact rejection. All epochs were normalized to a $100 \mathrm{~ms}$ baseline period from -201 to $-101 \mathrm{~ms}$ relative to visual stimulus onset, ending $1 \mathrm{~ms}$ before the time of the first TMS pulse: in this ERP analysis, the event that the potentials should be related to is the onset of the TMS.

The LRP is time-locked to a stimulus that requires a response, and measures lateralized motor preparatory activity (Fig. 3) (Coles, 1989). Following Coles (Coles, 1989; Coles et al., 1995), it was calculated as follows: $\mathrm{LRP}=\left[\right.$ mean $(\mathrm{C} 4-\mathrm{C} 3)_{\text {left-hand movement }}+$ mean $(\mathrm{C} 3-\mathrm{C} 4)_{\text {right- }}$ hand movement $] / 2$. C3 and $\mathrm{C} 4$ are the electrode positions above the left and the right lateral motor cortex. In other words, the EEG recorded over motor cortex contralateral to the moved hand (e.g., C4 is contralateral to the left hand) is refined by subtracting from it the waveform recorded simultaneously over the ipsilateral electrode (e.g., C3). This makes explicit the lateralized negativity that precedes a movement (Kornhuber and Deecke, 1965). An analogous subtraction is performed for data recorded on trials on which the right hand is moved, but now the waveform recorded at $\mathrm{C} 4$ is subtracted from the waveform at $\mathrm{C} 3$. The results of this subtraction for each hand are averaged together, not only to give a hemisphere-independent measure of motor preparation but also to subtract away any nonmotor asymmetrical activity. The output of this procedure therefore incorporates recordings made from both motor cortices, but it only includes activity that is both lateralized and that varies with the side of the to-be-executed movement (Fig. 3). The LRP reflects predominantly activity in the primary motor cortices (Arezzo and Vaughan, 1975; Brunia, 1988; Requin et al., 1988) and is a measure of partial response activation (i.e., it reflects the currently planned response, even if that response is not eventually executed). This means that the initial preparation of an incorrect movement on an incongruent trial is apparent as a positivity in the LRP, although the initial (incorrect) plan is

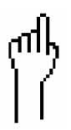

a

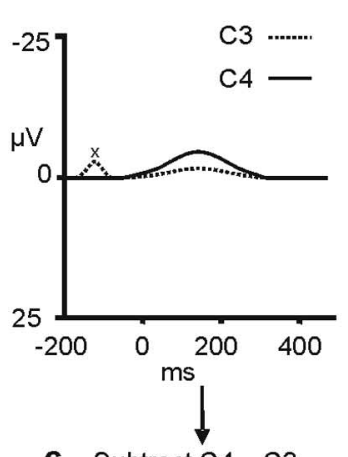

C Subtract $\mathrm{C} 4-\mathrm{C} 3$

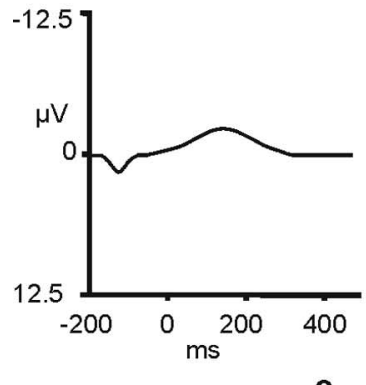

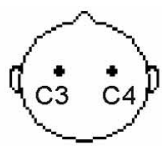<smiles>CCCCCC1CCCCC1</smiles>

b Right Hand

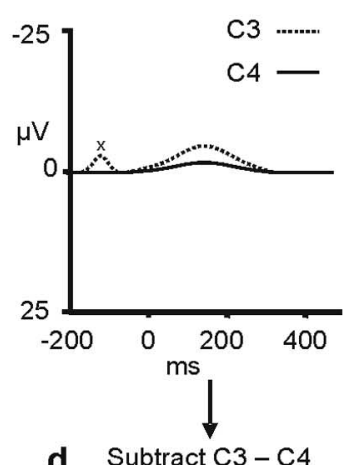

d Subtract $\mathrm{C} 3-\mathrm{C} 4$
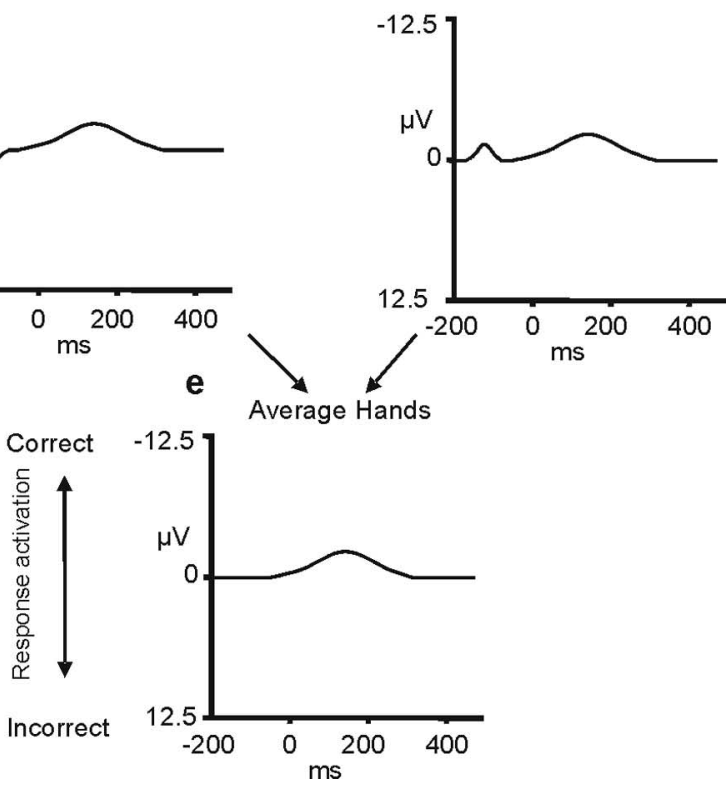

Figure 3. Derivation of the LRP. The top row of figures $(\boldsymbol{a}, \boldsymbol{b})$ show the event-related potentials recorded at the $\mathrm{C} 3$ and $\mathrm{C} 4$ electrodes over the left and right motor cortex (dotted and continuous lines, respectively) when left-hand $(\boldsymbol{a})$ and right-hand $(\boldsymbol{b})$ responses are made. As can be seen, there is a signal in the event-related potential recorded from each hemisphere and it is more negative (upward direction) over the motor cortex contralateral to the hand that moves. In the next stage $(\boldsymbol{c}, \boldsymbol{d})$, a difference wave is derived that reflects the difference in the signal recorded from the $\mathrm{C} 3$ and $\mathrm{C} 4$ electrodes ( $\mathrm{C} 4$ - C3 for the left-hand movements in c; $\mathrm{C} 3-$ ( 4 for the right-hand movements in $\boldsymbol{d}$ ). These two difference waves are then averaged to form the LRP (e). The LRP derivation removes any activity unrelated to the process of movement preparation that is not lateralized with respect to the moving hand (for example, the deviation in the signal labeled " $x$ "). This figure was adapted from Coles (1989). A negative LRP (deflection in the upward direction) indicates greater activation of the correct as opposed to the incorrect response representation.

canceled and only the correct movement is performed. Because the LRP is a difference wave, rather than a standard single-condition eventrelated potential, it is particularly well suited as an index of M1 activity in a TMS experiment. Any effects of TMS unrelated to action selection are common to all the component waveforms from which it is composed and so they are cancelled out by the subtraction of one component from the other (Fig. 3).

We examined how congruence and TMS affected motor preparatory activity by systematically averaging the amplitude of the LRP for each condition across $20 \mathrm{~ms}$ time bins from 120 to $400 \mathrm{~ms}$ after presentation of 
the visual stimulus (this starts $20 \mathrm{~ms}$ after the last TMS pulse in each train). Although response execution may not be completed by $400 \mathrm{~ms}$, subjects' RTs may exceed $400 \mathrm{~ms}$, the response selection and preparation processes indexed by the LRP are normally complete by this time, and this was also true in the present experiment. Effects of congruence, TMS, and their interaction were tested for with a repeated-measures ANOVA. To correct for multiple comparisons, main effects were only considered as significantly different if lasting for at least two consecutive time bins.

Interactions were only considered significant if occurring at the same time as such consecutive main effects, or if lasting for two consecutive time bins themselves. Repeated-measure ANOVAs were also used to examine behavioral measures including RTs and errors. Three two-level factors were considered: congruence (congruent or incongruent), TMS (TMS trial or no-TMS trial), and hand (trials in which the correct response was to be made with the right hand or the left hand).

\section{Experiment 2: control-site TMS}

The results of experiment 1 demonstrated that the TMS had a specific effect inasmuch as it disrupted performance on the incongruent conflict trials but not on the congruent trials. It is, however, important to demonstrate not just that TMS effects are specific to a particular cognitive task, or a particular type of trial within a cognitive task, but it is also necessary to show that the effect of the TMS is specific to a particular region of cortex. If, however, the effect of the dMFC TMS is only a consequence of any visual or tactile sensation associated with the TMS, then a similar effect on the LRP would be expected after TMS to a control site.

Participants. Sixteen right-handed subjects (nine females; mean age, 25; range, 18-27) participated in the separate control site experiment, designed to rule out any possible confounds from acoustic or somatosensory TMS-related artifacts. Two of the subjects had previously participated in experiment 1.

$E E G$ recording. The electrode montage used was the same apart from electrode $\mathrm{CPZ}$ replacing $\mathrm{PZ}$ and electrode $\mathrm{OZ}$ replacing $\mathrm{PO}$, to accommodate the TMS coil. As in the active site, data from two subjects were rejected because not enough trials survived artifact rejection, and the mean number of trials subject per condition was the same (45).

TMS. TMS was delivered over a control site $5 \mathrm{~mm}$ lateral, to the left, of electrode position PZ-POZ, overlying parieto-occipital cortex. In a subset of eight subjects, Brainsight frameless stereotactic registrations of individuals' structural MRIs into standard space (Fig. $2 b$ ) verified that the control sites clustered tightly in parieto-occipital cortex (mean, $x=$ $-6, y=-81, z=52$ ). The position adjacent to PZ-POZ was chosen as the control site for three reasons. First, it was approximately the same distance from the critical C3 and C4 electrode positions as was the dMFC position adjacent to FCZ. Second, it meant that TMS in both the control and $\mathrm{dMFC}$ conditions was targeted $5 \mathrm{~mm}$ to the left of the midline. Third, it meant that TMS was applied over a region that has not been associated with the mediation of response conflict. Blood oxygenation leveldependent (BOLD) signal changes have been recorded in the parietal cortex during the performance of tasks involving a high degree of response conflict, but only at more anterior locations (Rushworth et al., 2001; Liston et al., 2006).

Task design and analysis. All aspects of the task design and analysis were the same as in experiment 1 .

\section{Results \\ Experiment 1: dMFC TMS \\ EEG}

The effect of congruence on motor preparation was evident in the EEG (Fig. 4). Congruence significantly modulated the LRPs between 220 and $360 \mathrm{~ms}\left(F_{(1,13)}\right.$ values $\geq 7.062$; $p$ values $\left.<0.05\right)$. The key result is that TMS also modulated this congruence effect from 180 to 220 and 260 to $280 \mathrm{~ms}$ (interaction of TMS and congruence; $F_{(1,13)}$ values $\geq 6.243$; $p$ values $<0.05$ ) with an additional marginal interaction during the 280-300 $\mathrm{ms}$ time bin $\left(F_{(1,13)}=3.866 ; p=0.07\right)$. Additional analysis showed that the interaction was primarily attributable to the significant difference

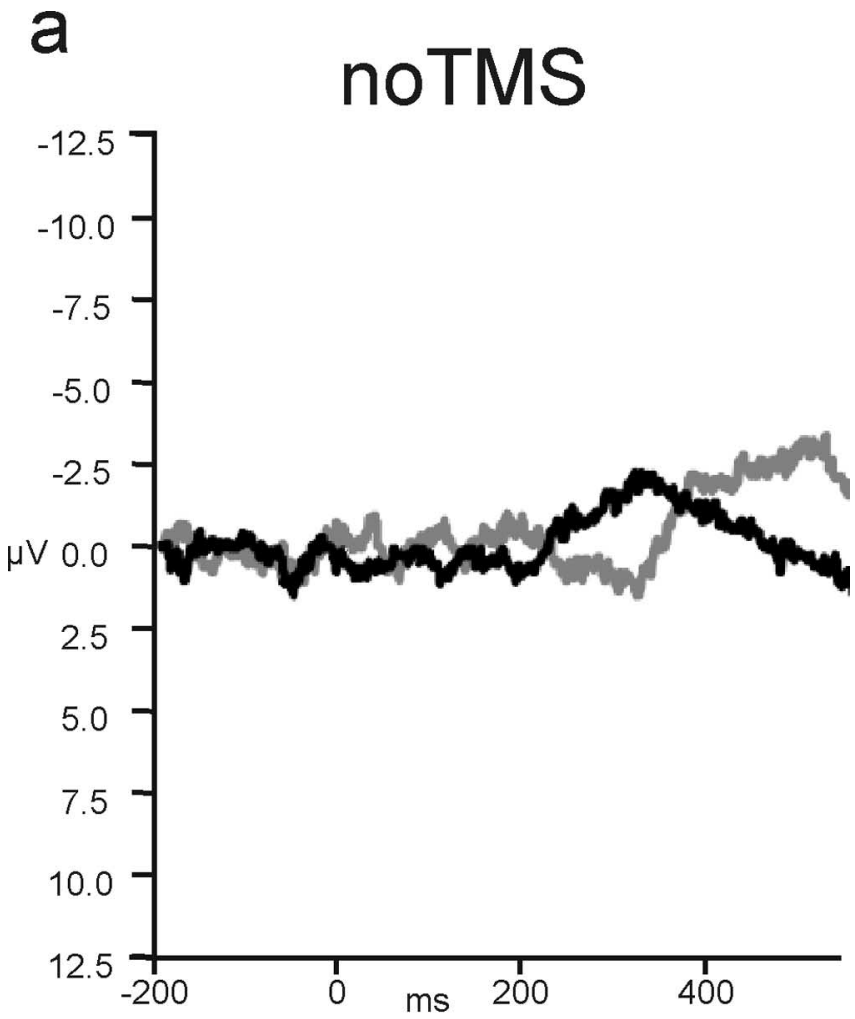

b

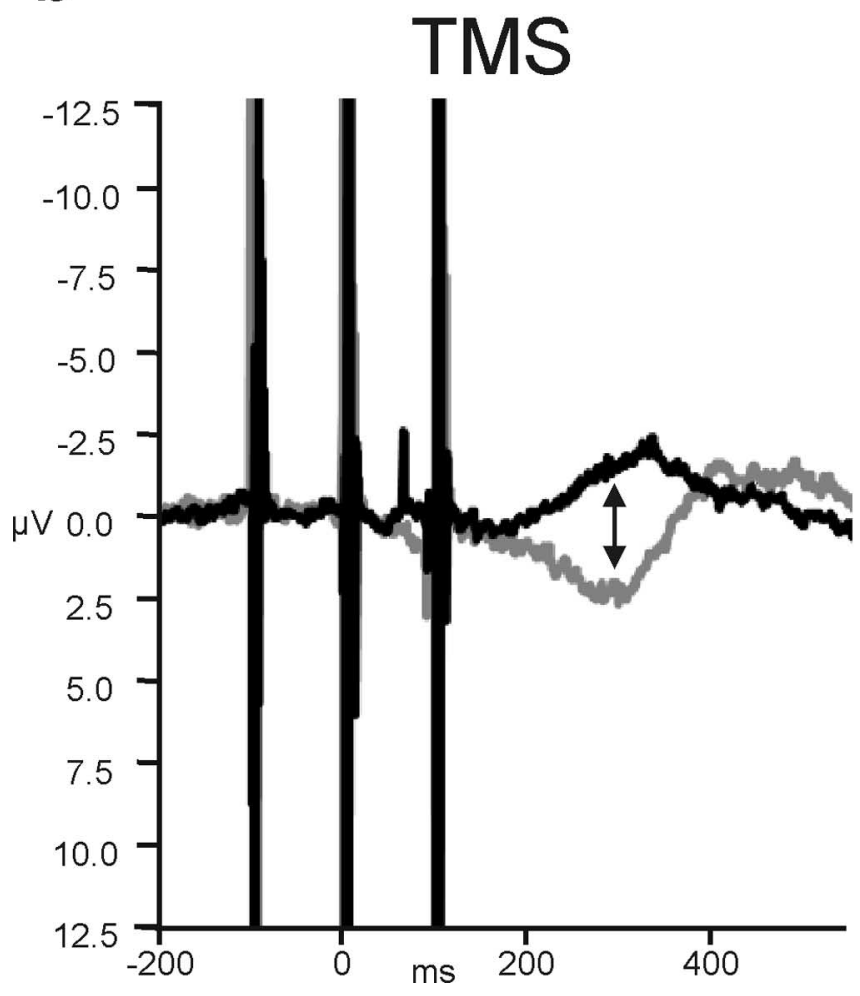

Figure 4. The effect of dMFC TMS on the LRP. $\boldsymbol{a}$, On no-TMS congruent trials (black), there was a clear negative deflection in the LRP, indicative of the preparation of correct responses, peaking at $\sim 300 \mathrm{~ms}$. On incongruent trials (gray), the waveform was, instead, displaced in the positive direction, associated with the preparation of the wrong response. The negative deflection associated with the correct response was delayed. $\boldsymbol{b}$, When dMFC TMS was applied, there was a significant increase in the difference between the waveforms recorded on congruent and incongruent trials starting at $180 \mathrm{~ms}$. This was attributable to dMFC TMS causing a positive deflection in the LRP on incongruent trials. Negative is plotted upward for the LRP. 
in the incongruence waveform on TMS and no-TMS trials from 180 to 220 and 260 to $280 \mathrm{~ms}\left(t_{(13)}\right.$ values $\geq 1.80$; $p<0.05$; onetailed test), whereas TMS-induced differences did not approach significance in the congruent condition $\left(t_{(13)}\right.$ values $\leq 0.730 ; p>$ 0.24 ; one-tailed test). TMS therefore had a differential effect on the motor preparatory activity indexed by the LRP, depending on the task condition, only modulating the LRP on incongruent trials (Fig. 4). Because negativity is associated with preparation of the correct response, the positive (downward) shift of the incongruent waveform by TMS is consistent with a greater activation of the incorrect response associated with the flanking distractor stimuli on incongruent trials. We also found that the difference between the congruent and incongruent waveforms lasted longer after dMFC TMS than on no-TMS trials. In the absence of TMS, the incongruent waveform was significantly different from the congruent waveform for $\sim 60 \mathrm{~ms}$ between the 280 and $320 \mathrm{~ms}$ time bins $(t>3.5$; df $=13 ; p<0.05)$. When dMFC TMS was applied the period of significant difference extended over $160 \mathrm{~ms}$ between the 200 and $340 \mathrm{~ms}$ time bins $(t>3.1$; df $=13 ; p<0.05)$.

All the analyses, including those just described, focused on only those trials preceded by a previous no-TMS trial to rule out any possible confounding influence of TMS from a previous trial. To further corroborate our conclusions, we also performed an additional analysis that focused only on those trials, both TMS and no-TMS, that had been preceded by a no-TMS congruent trial. Gratton et al. (1988) have shown that additional control is exerted over response selection processes on trials that follow previous conflict trials. The role of the lateral prefrontal cortex in exerting such across-trial long-latency control has been emphasized (Botvinick et al., 2004). If, however, the dMFC has a shortlatency within-trial influence on response selection even on the first conflict trial encountered, then dMFC TMS should also increase the LRP difference for congruent-incongruent trials that follow previous congruent trials. We examined data pooled from the time bins $180-220$ and 260-300, because it was in these time bins that there were interactions between congruence and TMS in the original data set. Both sets of time bins continued to show interactions between congruence and TMS as before $\left(F_{(1,13)}\right.$ values $>5.1$; $p$ values $<0.05$ ) (Fig. 5).

\section{Hand-specific ERP effects of dMFC TMS}

Because the dMFC was applied over the left hemisphere, an analysis was also conducted to test whether there were any lateralized effects on ERP components related to response selection; if the $\mathrm{dMFC}$ is concerned with the resolution of response conflict instead or in addition to conflict detection, then it might be expected that ERPs on right-hand response selection trials would be most affected by the dMFC TMS. Rather than examining the LRP, which reflects processes related to the selection of both right and left responses, this analysis focused on the difference between the congruent and incongruent ERPs recorded at the left- and right-hemisphere electrodes $\mathrm{C} 3$ and $\mathrm{C} 4$ separately, and comparing left- and right-hand response trials. Congruence again reliably modulated the ERPs starting from $340 \mathrm{~ms}$ until the end of the epoch at $400 \mathrm{~ms}\left(F_{(1,13)}\right.$ values $\geq 10.9 ; p$ values $\left.<0.05\right)$. There was also a main effect of hemisphere $(320-400 \mathrm{~ms})\left(F_{(1,13)}\right.$ values 27.2 ; $p$ values $<0.05)$ and an effect of TMS at the start of the epoch $(120-240 \mathrm{~ms})\left(F_{(1,13)}\right.$ values $\geq 5.1 ; p$ values $\left.<0.05\right)$. In addition to interactions of congruence with hemisphere $(260-$ $360 \mathrm{~ms})\left(F_{(1,13)}\right.$ values $\geq 9.5$; $p$ values $\left.<0.05\right)$ and with hand $(160-260 \mathrm{~ms})\left(F_{(1,13)}\right.$ values $\geq 6.4 ; p$ values $\left.<0.05\right)$, there was a key four-way interaction between hemisphere, hand, congruence, and TMS from 220 to $360 \mathrm{~ms}\left(F_{(1,13)}\right.$ values $\geq 4.8$; $p$ val-
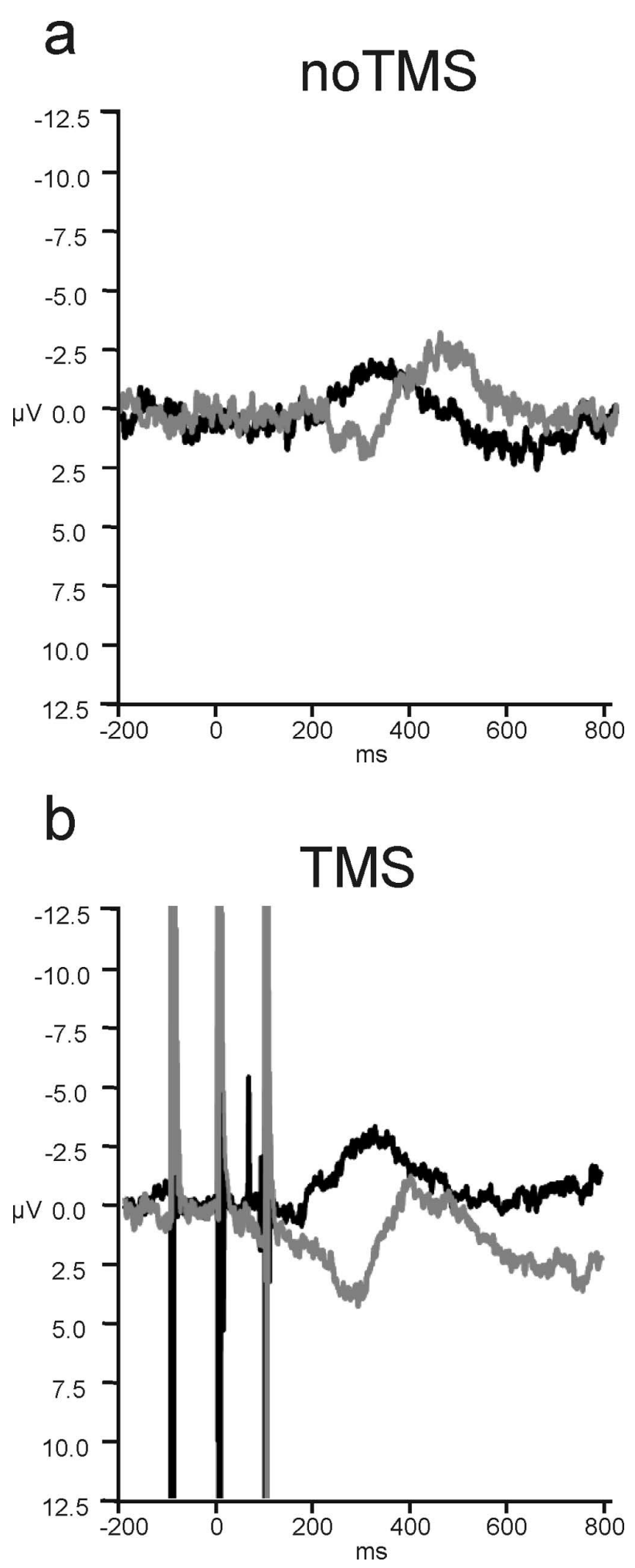

Figure 5. $\mathrm{dMFC}$ TMS effects on the LRP on trials preceded by congruent no-TMS trials. $\boldsymbol{a}, 0 \mathrm{n}$ no-TMS trials, the congruence effect was maintained even when only looking at that subset of trials after congruent trials. $\boldsymbol{b}$, After TMS, the congruence effect was still increased and this effect was still limited to the incongruent trials.

ues $<0.05$ ). This four-way interaction was attributable to a threeway interaction between hemisphere, congruence, and TMS only being evident for right-hand responses $(160-360 \mathrm{~ms})\left(F_{(1,13)}\right.$ values $\geq 5.3$; $p$ values $<0.05$ ) and not left-hand responses. In other 

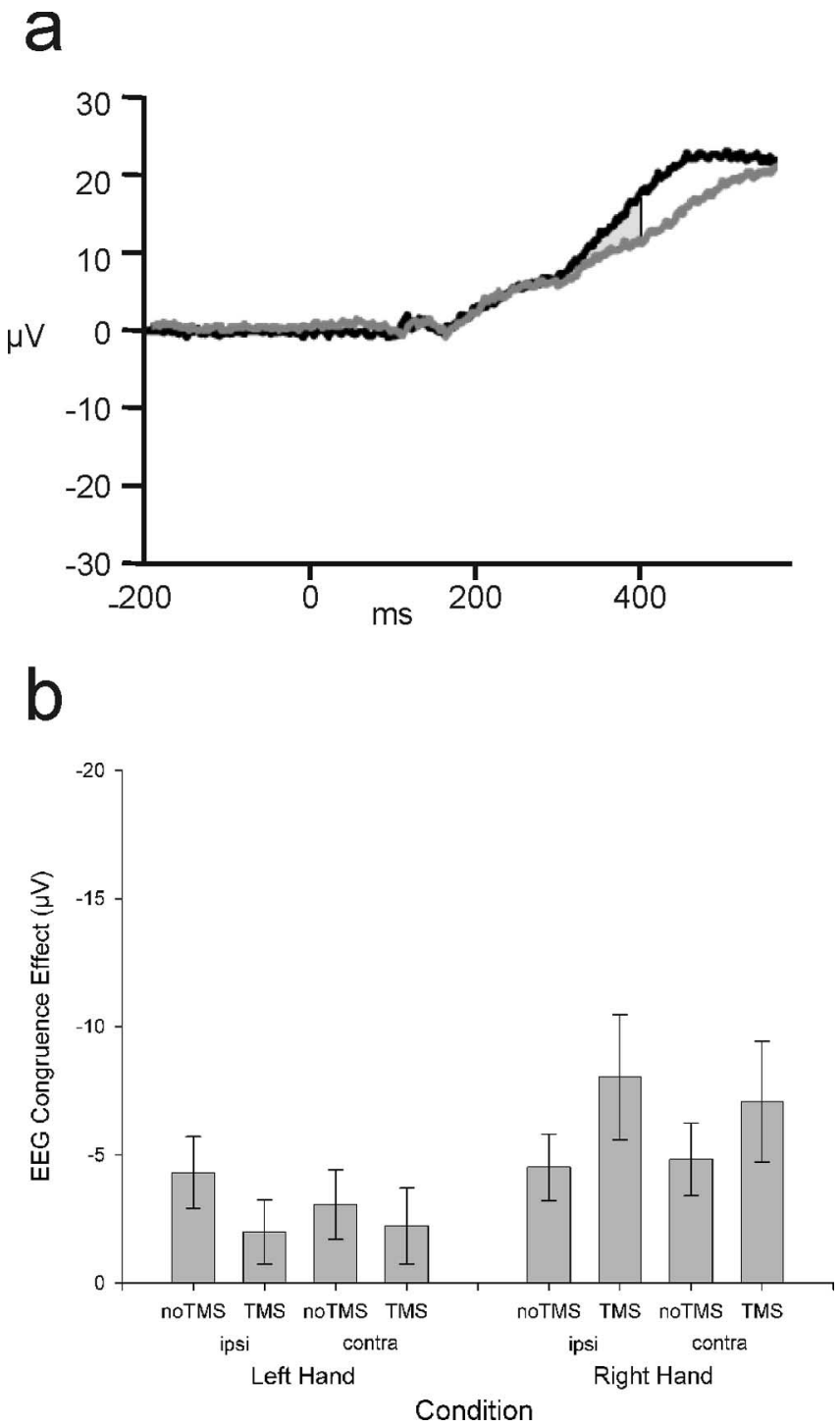

Figure 6. Hand-specific effects. $\boldsymbol{a}$, Example ERP data (without LRP derivation; negative plotted downward) recorded from electrode $(3$ on no-TMS right-hand response trials showing divergence of congruent (black) and incongruent (gray) waveforms from $340 \mathrm{~ms}$. $\boldsymbol{b}$, The congruence effect (incongruent minus congruent between 340 and $400 \mathrm{~ms}$ ) is plotted separately for left- and right-hand response trials (left and right of figure) and separately for the hemisphere ipsilateral (ipsi) and contralateral (contra) to the response hand. dMFC TMS specifically increased the congruence effect on right-hand trials regardless of hemisphere. Error bars indicate SEM.

words, left dMFC TMS modulated the congruency effect on right-hand response selection-related ERPs, but it did not modulate the congruency effect on left-hand trials (Fig. 6) Such a hand-specific effect provides additional support for a role of dMFC in conflict resolution rather than just conflict detection. There was no effect of hemisphere for right-hand responses [i.e., we did not find evidence here that either one of the correct (left hemisphere) or incorrect (right hemisphere) motor plans were being disrupted more than the other].

Behavioral results

The primary aim of the current experiment was to examine the physiological impact of dMFC TMS rather than its behavioral impact. Previous dMFC TMS studies that have reported behavioral effects have often used trains of at least five pulses rather than the three pulse train used here (Hadland et al., 2001; Rush-
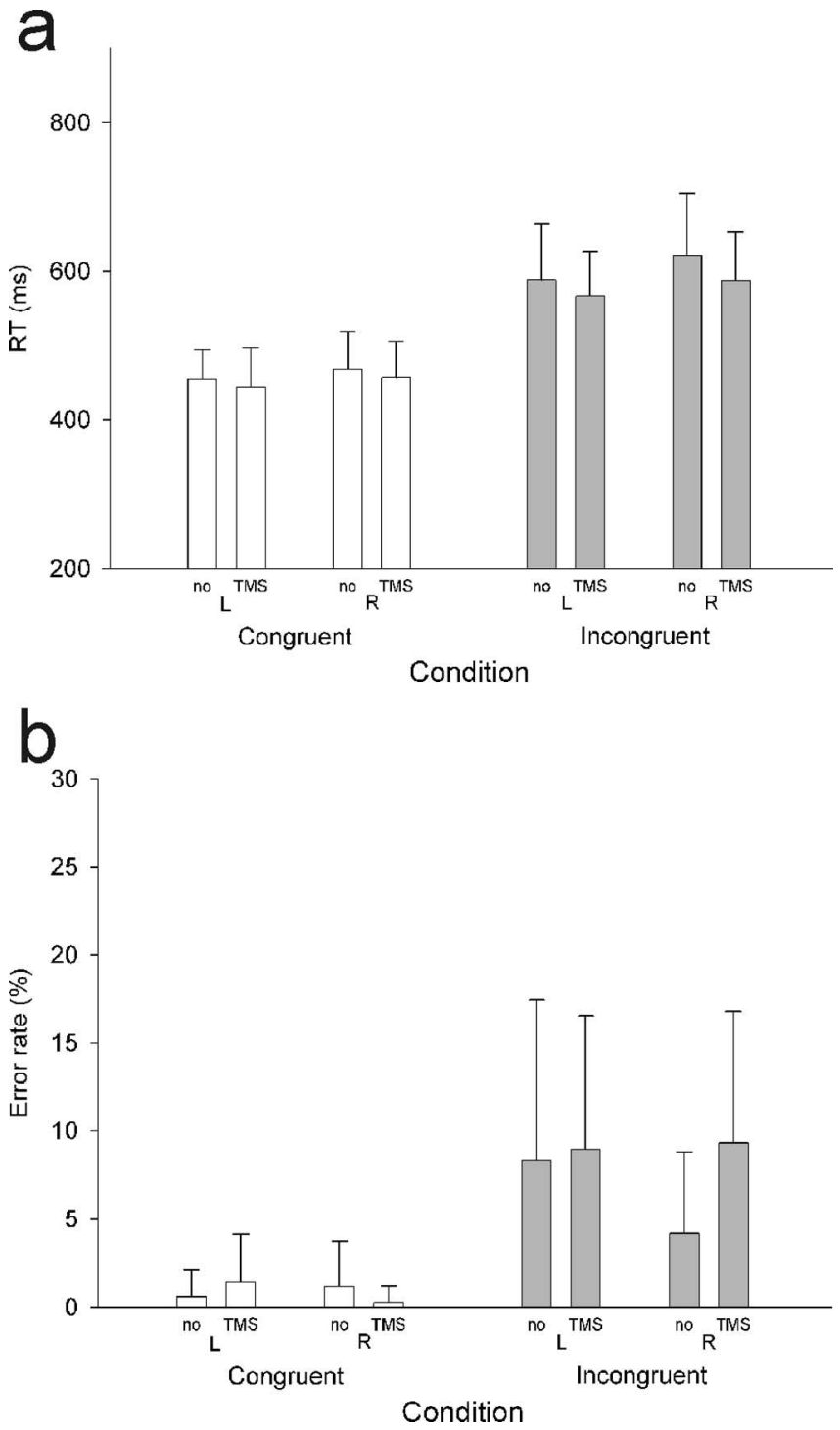

Figure 7. dMFC behavioral results. Column heights indicate group mean RTs, and error bars indicate the SE of the estimate of means. RTs were longer $(\boldsymbol{a})$ and errors were more common $(\boldsymbol{b})$ on incongruent than on congruent trials. There was a significant interaction between the effect of dMFC TMS, hand, and congruence on error rates. There was a significant difference between dMFC TMS and no-TMS error rates only on right-hand response incongruent trials.

worth et al., 2002; Kennerley et al., 2004). Nevertheless, it was noted that target and distractor congruence had a significant impact on response selection (Fig. $7 a$ ): although accuracy was high (>90\%), congruence effects were apparent on error rates (Fig. $7 b)$, with more errors on incongruent $(9 \%)$ than on congruent $(1 \%)$ trials (main effect of congruence; $F_{(1,13)}=27.6 ; p<0.001$ ). There was a three-way interaction between congruence, hand, and TMS $\left(F_{(1,13)}=6.7 ; p<0.05\right)$ : delivery of TMS over the left $\mathrm{dMFC}$ interacted with the congruence effect (incongruent minus congruent error rates) on trials requiring the contralateral righthand response (TMS, 9.1\%; no-TMS, 3.0\%; $t=-3.4 ; p<0.01$ ) but not the ipsilateral left-hand response $(t=0.09 ; p=0.9)$. It was noted, however, that subjects appeared to find incongruent trials that required a left-hand response more difficult than those requiring a right-hand response $\left(t_{(13)}=3.458\right.$; $p<0.05$; onetailed $t$ test). We therefore compared the error rates on incongruent trials with and without TMS. There was a significant increase in error rates on right-hand response incongruent trials when 


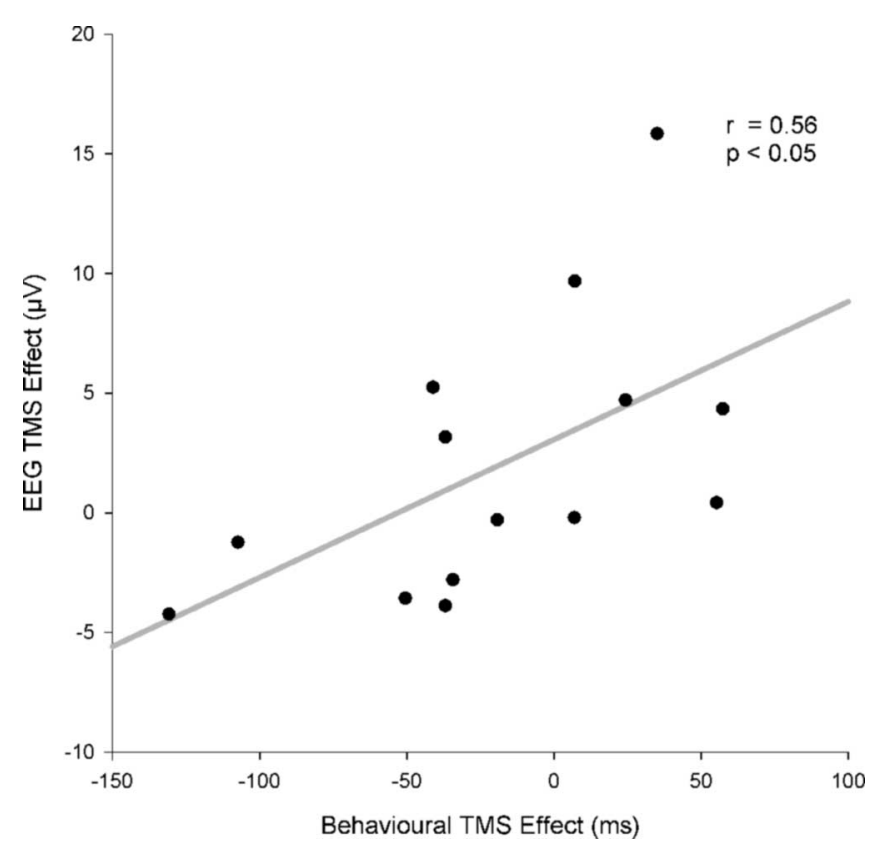

Figure 8. Positive correlation between dMFC TMS effects on behavior, as indexed by RT and the LRP. The effect of dMFC TMS on conflict resolution was calculated for both behavior and the LRP as TMS incongruent-congruent $_{- \text {no-TMS }}$ incongruent- congruent. Subjects who showed the strongest effects of dMFC TMS on the behavioral measure of conflict also showed the strongest effects on the LRP measure of conflict.

TMS was delivered (TMS, 9.3\%; no-TMS, 4.2\%; $t=-2.7 ; p<$ 0.05 ), but there was no similar effect on left incongruent trials nor on left or right congruent trials (all $p$ values $>0.2$ ).

Congruence effects were also apparent in the RTs that were markedly slower on incongruent trials than on congruent trials (main effect of congruence; $F_{(1,13)}=117.3 ; p<0.001$ ). Again, a difference between trials performed with the two hands was noted; subjects were slower on right-hand response trials than on left-hand response trials (main effect of hand; $F_{(1,13)}=6.3 ; p<$ 0.05 ) and were faster, in general, on TMS trials than no-TMS trials (main effect of TMS; $F_{(1,13)}=9.2 ; p<0.01$ ). This may be attributable to the general alerting effect of TMS (Marzi et al., 1998).

A between-subject correlation analysis was also conducted to test for any relationship between the impact that the dMFC TMS had on behavior and on the LRP (Fig. 8). The impact of dMFC TMS on the LRP was indexed using the main effect of our study, the size of the difference between congruent and incongruent waveforms after TMS compared with without TMS (TMS incongruent-congruent $_{\text {- no-TMS }}$ incongruent-congruent $)$. The LRP amplitudes were measured at the peak difference between TMS congruent and incongruent waveforms, indicated with an arrow in Figure $4(340 \mathrm{~ms})$. The effect of dMFC TMS on behavior was measured using the identical comparison (TMS $_{\text {incongruent-congruent }}-$ no-TMS incongruent-congruent $_{\text {) }}$ but using RT data rather than LRP amplitudes. A significant correlation was found between the effect of dMFC TMS on the LRP and on RT (Pearson's $r=0.57 ; N=14 ; p<0.05$ ). A nonparametric correlation confirmed that the correlation was robust and could not be attributed to any single outlier (Spearman's $r=0.61 ; N=$ $14 ; p<0.05)$. Subjects in whom the TMS caused the greatest increase in the congruence effect as evident in the LRP also showed the greatest increase in the corresponding congruence effect measured with RTs (Fig. 8).

\section{Experiment 2: control-site TMS}

EEG recording

Congruence modulated the LRP from 200 to $340 \mathrm{~ms}\left(F_{(1,13)}\right.$ values $\geq 4.761$; $p$ values $<0.05$ ), following the same pattern as experiment 1 . Unlike in experiment 1 , however, control-site TMS did not modulate this congruence effect: although there was a slight deviation in both congruent and incongruent waveforms that resulted in a main effect of TMS from 280 to $400 \mathrm{~ms}\left(F_{(1,13)}\right.$ values $\geq 4.838$; $p$ values $<0.05$ ), there was no interaction between TMS and congruence in any time bins (Fig. 9). In addition, additional tests showed that, unlike the case with dMFC TMS, control TMS did not induce any difference in the incongruent waveform (all $p$ values $>0.2$; one-tailed test) (Fig. 10).

Another analysis focused just on those trials that had been preceded by a previous congruent trial but once again found no effects reaching significance after control site TMS $\left(F_{(1,13)}\right.$ values $<0.3$; $p$ values $>0.6$ ) .

Additionally, we compared the TMS-induced effects on incongruent trials in experiments 1 and 2. dMFC TMS caused a significantly greater deflection on incongruent trials than did control TMS on most time bins between 180 and 360 ms (200$240 \mathrm{~ms}, 260-300 \mathrm{~ms}$, and at $340 \mathrm{~ms}$; all $t$ values $\geq 2.06$; $p$ values $<0.05)$ with additional marginal effects in three intervening time bins $\left(180,240\right.$, and $320 \mathrm{~ms} ; t_{(24)}$ values $\geq 2.1 ; p$ values $\leq 0.089$ ).

There were also no significant hand specific effects reminiscent of those seen in the dMFC TMS experiment (Fig. 6). There were no interactions between TMS, hemisphere, and congruence apparent for either left-hand or right-hand responses in any time bins $\left(F_{(1,13)}\right.$ values $\leq 1.9$; $p$ values $\left.>0.19\right)$ (Fig. 11) when the C3 and C4 ERP signals were compared for left- and right-hand responses on congruent and incongruent trials.

\section{Behavioral results and EEG-behavior correlations}

As in the dMFC TMS experiment, the incongruence-congruence manipulation affected performance, with subjects making more errors on incongruent trials (main effect of congruence; $F_{(1,14)}=$ 38.0; $p<0.0001$ ) (Fig. 12). Unlike dMFC TMS, however, the control site TMS exerted no significant effect on behavior.

Both the dMFC TMS site and the control site were $0.5 \mathrm{~cm}$ from the midline and situated over the left hemisphere. Any effect of the control site TMS was therefore expected to be maximal in the contralateral hand just as had been the case for the dMFC TMS. A comparison of the effect of left dMFC TMS and control site TMS on right-hand responses demonstrated a significant interaction of TMS, site, and congruence $\left(F_{(26)}=11.0 ; p<0.05\right)$. The TMS-induced change in errors was significantly greater when TMS was applied over dMFC as opposed to the control site $(t=3.0 ; \mathrm{df}=26 ; p<0.05)$. There was no effect of TMS on congruent trials in either dMFC or control site TMS experiments (all $p$ values $\geq 0.24$ ).

RTs were slower on incongruent than congruent trials (main effect of congruence; $F_{(1,14)}=53.5 ; p<0.001$ ) and faster on TMS than no-TMS trials $\left(F_{(1,14)}=8.8 ; p<0.05\right)$. There was also a trend toward a main effect of hand, with worse performance on righthand response trials $\left(F_{(1,14)}=3.0 ; p=0.105\right)$, but there were no interactions between hand and congruence.

Finally, we examined the relationship between the control site TMS-induced behavioral and electrophysiological effects. Unlike in the case of dMFC TMS, additional analyses were unable to find any correlation between the effect of control-site TMS on behavior, as indexed by RT, and LRPs (Spearman's $r=0.26$; $n=14$; $p=0.4)$. 

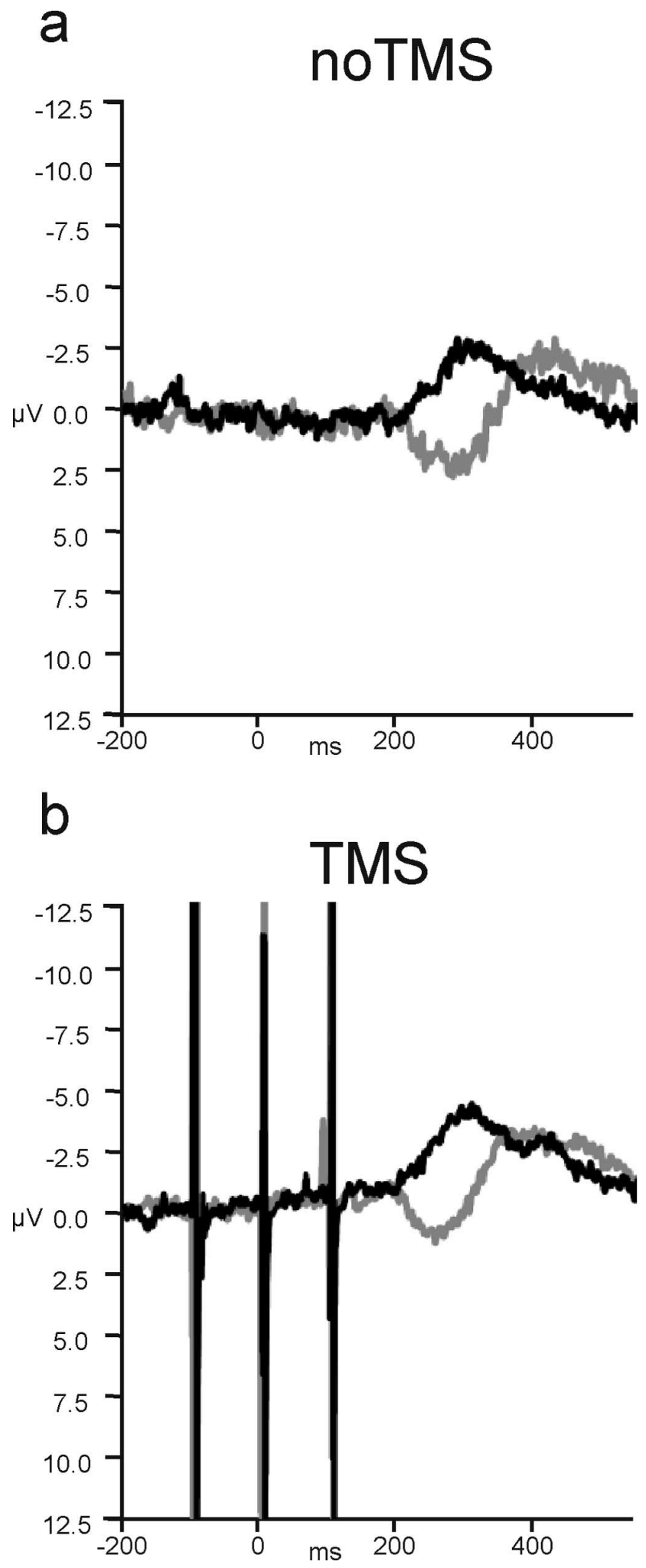

Figure 9. The effect of control TMS on the LRP. $\boldsymbol{a}$, Congruence modulated the LRP on no-TMS trials as in experiment $1 . \boldsymbol{b}$, Control TMS did not affect how congruence modulated the LRP, only causing a slight increase in the negative LRP deflection regardless of condition.

In summary, the influence of factors such as congruence and hand were similar to the effects recorded in experiment 1 in which TMS was applied to the dMFC site. In experiment 2, however, control site TMS exerted no significant effect on behavior (Fig. 12).

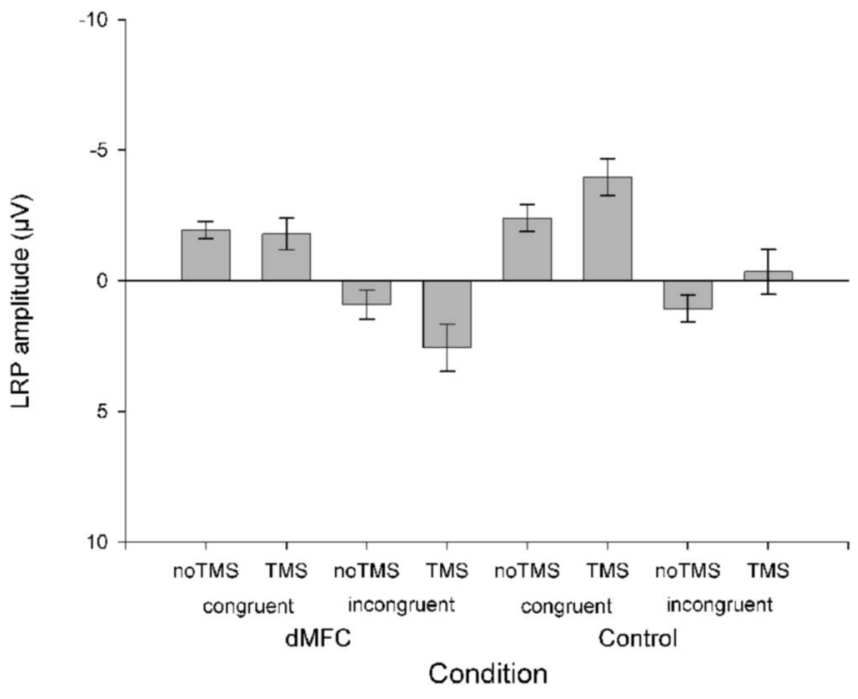

Figure 10. LRP amplitudes summarized for $\mathrm{dMFC}$ and control site experiments. TMS only increased the LRP amplitude (measured at $300 \mathrm{~ms}$ after cue onset) when applied to dMFC during incongruent trials. Negative is plotted upward. Error bars indicate SEM.

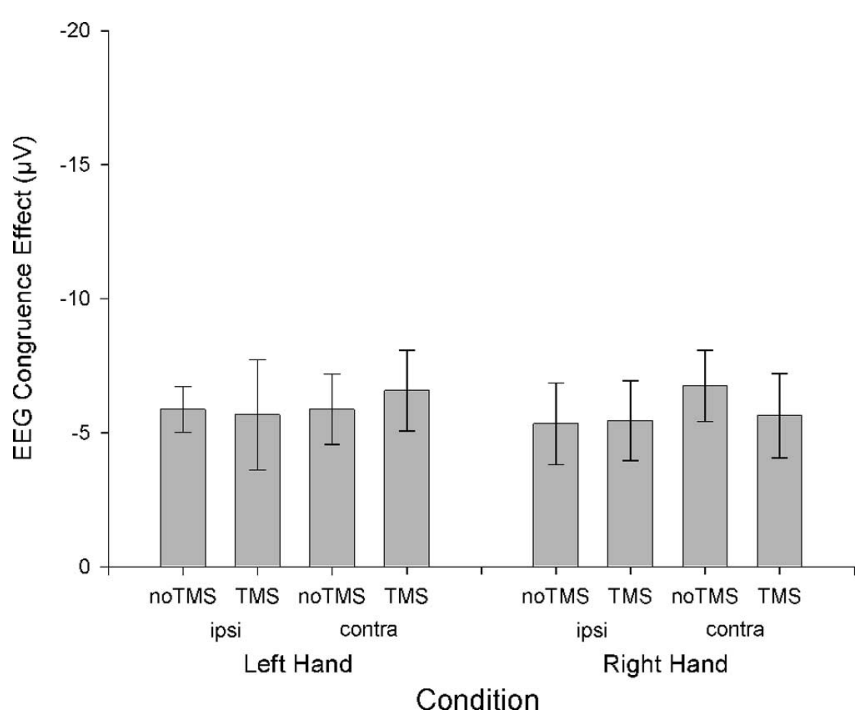

Figure 11. Control TMS hand-specific effects. After control TMS, there was no evidence of modulation of the congruence effect as had been found with dMFC TMS (Fig. 6). Error bars indicate SEM. ipsi, Ipsilateral; contra, contralateral.

\section{Discussion}

dMFC TMS interfered with the normal modulation of motor cortical activity by conflict in a flanker task (Eriksen and Eriksen, 1974), shifting the LRP on incongruent trials in the positive direction, associated with preparation of the incorrect response (Fig. 4). There was a positive correlation between the effect of dMFC TMS on LRP measures of conflict, which reflect processes related to both left- and right-hand response selection, and on RT measures of conflict averaged across hands (Fig. 8). The dMFC TMS was applied over the left hemisphere (Fig. 2a) and particularly affected conflict-related ERP differences for contralateral right-hand responses (Fig. 6). Error rates were also only significantly increased on right-hand incongruent trials (Fig. 7). No similar behavioral (Fig. 12) or ERP effects were seen with control TMS (Fig. 9). Our results indicate that one mechanism by which 


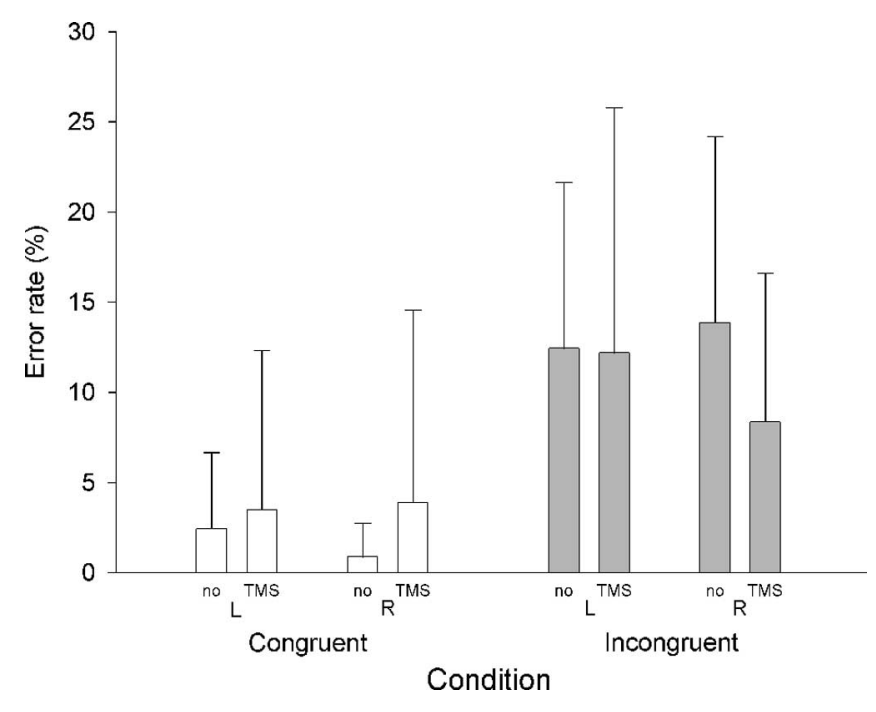

Figure 12. Control TMS behavioral results. Column heights indicate group means and error bars indicate the SE of the estimate of means. Errors were more common on incongruent than on congruent trials, but there was no significant impact of the TMS at the control site.

dMFC resolves response conflict is by modulating primary motor cortical activity.

dMFC TMS has a short-latency within-trial effect on response selection in motor cortex. It has previously been emphasized that $\mathrm{dMFC}$ detects conflict and then instructs other brain structures to exert greater control over response selection on subsequent trials (Botvinick et al., 2004; Rushworth et al., 2004). Rather than directly test this hypothesis, we tested whether the dMFC, in addition or instead, has a short-latency role in actively resolving response conflict within the same single trial that first engenders conflict. The significant impact of dMFC TMS was still apparent even when comparing just those trials preceded by a congruent trial (Fig. 5).

Microstimulation of $\mathrm{dMFC}$, in the supplementary eye field (Stuphorn and Schall, 2005) or pre-SMA (Isoda and Hikosaka, 2007), affects tasks requiring the inhibition of a planned saccade to execute an alternative saccade. We extend this work by investigating human dMFC during the Eriksen flanker paradigm, which has been explored in previous human neuroimaging studies (Botvinick et al., 2004; Rushworth et al., 2004). It might be argued that the conflict occurring in monkey studies is not representative of human neuroimaging studies because there is no simultaneous exposure to stimuli instructing multiple actions, but the present results confirm the importance of dMFC for within-trial conflict resolution. The hand-specific effects seen here are also consistent with the lateralized effects of microstimulation on conflict and with the emphasis such studies have placed on the executive role of $\mathrm{dMFC}$ in the resolution, rather than just the detection, of conflict.

The LRP effects illuminate the mechanism by which dMFC functions, because the LRP measures activity in lateral motor cortex, particularly primary motor cortex (M1) (Arezzo and Vaughan, 1975; Brunia, 1988; Requin et al., 1988; Coles, 1989). A negative LRP deflection reflects preparation of the correct movement, whereas a positive deflection demonstrates that a plan for the incorrect movement has been partially activated within the M1 controlling the inappropriate hand (Fig. $3)$. The time at which dMFC TMS modulated the LRP included the time at which congruence normally modulates the
LRP: TMS affected the LRP congruence effect from $180 \mathrm{~ms}$ after visual stimulus onset, well before movement execution, until $300 \mathrm{~ms}$. The dMFC TMS-induced positive LRP deflection on incongruent trials is therefore consistent with the concomitant behavioral impairment. The incongruent flanker stimuli activated plans for both correct and incorrect movements, and to effectively resolve this conflict the dMFC appears to normally enhance the signal representing the correct movement in M1. dMFC TMS disrupts this normal top-down modulatory function and so the conflict is unresolved for longer, leading to prolonged motor cortical preparatory activity corresponding to each of the alternative responses (Figs. 4, 6 ) and an increased likelihood of slow responding and errors (Figs. 7, 8).

Although the dMFC TMS induced a short-latency modulation in motor activity, this probably occurred by an indirect anatomical route. The dMFC TMS was centered over pre-SMA (Fig. 2a). Although spread of induced current to adjacent brain areas is possible, studies using a similar coil have reported an effective resolution of approximately a centimeter (Brasil-Neto et al., 1992; Walsh and Cowey, 2000) and the effect of dMFC TMS can be distinguished from that of adjacent dorsal premotor cortex (Rushworth et al., 2002; Kennerley et al., 2004b). Although pre-SMA is not directly interconnected with M1 or spinal cord (Luppino et al., 1993; He et al., 1995), it contains neurons that respond when hand movements are made (Fuji et al., 2001) and it is interconnected with M1 through a multisynaptic pathway (Miyachi et al., 2005). It is realistic to assume that dMFC TMS could impact on M1 activity via a multisynaptic pathway. For example, the application of cerebellar TMS conditioning pulses can influence the response of M1 to a test TMS pulse (Werhahn et al., 1996). The importance of subcortical areas in mediating the influence of dMFC TMS on M1 cannot be ruled out, and there has been interest in the role of the subthalamic nucleus and opercular frontal regions in inhibiting movements (Aron and Poldrack, 2006). Previous work emphasizing a role for dMFC in conflict detection (Botvinick et al., 2004) has suggested that conflict resolution occurs via lateral prefrontal regions that then exert greater control over response selection on the next trial. With the limited electrode montage in the present experiment, it was not possible to test whether lateral prefrontal regions also mediated short-latency within-trial conflict resolution, but neuroimaging experiments have not emphasized their activation when the first high-conflict trial is encountered (Botvinick et al., 2004).

The control TMS and AMFC TMS sites were identical distances from the midline, equating for any lateralized TMSrelated somatosensory or acoustic artifacts on EEG or behavior. The control site lay over a region of parieto-occipital cortex medial and posterior to parietal regions recruited by conflict and task-switching manipulations (Rushworth et al., 2001; Braver et al., 2003; Liston et al., 2006). Although control TMS did not disrupt modulation of the LRP by congruence, there was a deflection of both congruent and incongruent baseline waveforms in the negative direction, associated with performance of the correct response. This may be attributable to the alerting somatosensory and acoustic artifacts associated with TMS (Marzi et al., 1998), because there was also a slight, but nonsignificant, behavioral improvement with control TMS.

dMFC may facilitate the representation of one action in the motor cortex at the expense of alternatives. The modulating in- 
fluence of dMFC over motor cortex reported here may also underlie dMFC involvement in other studies. For example, previous neuroimaging (Deiber et al., 1991; Frith et al., 1991; Lau et al., 2004; Nachev et al., 2005), lesion (Thaler et al., 1995), and TMS (Hadland et al., 2001) reports have emphasized dMFC activity during "free selection" tasks in which the agent must choose between all currently relevant and competing action plans in the absence of any instruction. A similar process may occur during task switching and when switching between action sequence subroutines or "chunks" when dMFC is also known to be important (Shima et al., 1996; Nakamura et al., 1998; Brass and von Cramon, 2002; Rushworth et al., 2002; Husain et al., 2003; Kennerley et al., 2004; Bunge et al., 2005; Forstmann et al., 2005; Crone et al., 2006a,b).

The resolution of response conflict may depend on a mechanism that is also needed when selecting between responses in other situations. The dMFC may be part of the response bottleneck that ensures that only a single response is selected at any time (Dux et al., 2006). Effects of dMFC TMS similar to those reported here might be attainable in a broad range of action selection paradigms.

Although the possibility of top-down control of one area by another has received considerable interest, it has proved difficult to investigate empirically in the human brain. Sakai and colleagues (Sakai et al., 2002; Sakai and Passingham, 2003) have examined correlations in the fMRI BOLD signal and shown that changes between prefrontal and other areas occur that lead to the facilitation of task-relevant representations. The present study complements such approaches by demonstrating the causal impact of a manipulation of the activity of one area on the activity recorded from a second area. It has previously been shown that TMS affects activity recorded from electrodes adjacent to the site of stimulation and from remote brain regions even in the context of a behavioral task (Fuggetta et al., 2006; Taylor et al., 2007), but this is the first instance of combined stimulation-recording to demonstrate changes in functional connectivity between two regions of human cortex on a subsecond timescale that are specific to a particular cognitive state (incongruent trials). The approach complements demonstrations that interregional interactions, as assessed by the application of paired TMS pulses, occur during specific, brief time periods with a duration of several tens of milliseconds (Koch et al., 2006; Silvanto et al., 2006). Measuring the time course of the consequences of interference at areas distal to the stimulation site provides a means with which to extend current knowledge of brain function and to start to assemble a systems view of how brain areas interact to contribute to cognitive function.

\section{References}

Arezzo J, Vaughan Jr HG, (1975) Cortical potentials associated with voluntary movements in the monkey. Brain Res 88:99-104.

Aron AR, Poldrack RA (2006) Cortical and subcortical contributions to Stop signal response inhibition: role of the subthalamic nucleus. J Neurosci 26:2424-2433.

Aron AR, Poldrack RA (2006) Cortical and subcortical contributions to Stop signal response inhibition: role of the subthalamic nucleus. J Neurosci 26:2424-2433.

Botvinick MM, Cohen JD, Carter CS (2004) Conflict monitoring and anterior cingulate cortex: an update. Trends Cogn Sci 8:539-546.

Brasil-Neto JP, McShane LM, Fuhr P, Hallett M, Cohen LG (1992) Topographic mapping of the human motor cortex with magnetic stimulation: factors affecting accuracy and reproducibility. Electroencephologr Clin Neurophysiol 85:9-16.

Brasil-Neto JP, McShane LM, Fuhr P, Hallett M, Cohen LG (1992) Topo- graphic mapping of the human motor cortex with magnetic stimulation: factors affecting accuracy and reproducibility. Electroencephologra Clin Neurophysiol 95:9-16.

Brass M, von Cramon DY (2002) The role of the frontal cortex in task preparation. Cereb Cortex 12:908-914.

Braver TS, Reynolds JR, Donaldson DI (2003) Neural mechanisms of transient and sustained cognitive control during task switching. Neuron 39:713-726.

Brunia CH (1988) Movement and stimulus preceding negativity. Biol Psychol 26:165-178.

Bunge SA, Kahn I, Wallis JD, Miller EK, Wagner AD (2003) Neural circuits subserving the retrieval and maintenance of abstract rules. J Neurophysiol 90:3419-3428.

Bunge SA, Wallis JD, Parker A, Brass M, Crone EA, Hoshi E, Sakai K (2005) Neural circuitry underlying rule use in humans and nonhuman primates. J Neurosci 25:10347-10350.

Coles MGH (1989) Modern mind-brain reading: psychophysiology, physiology, and cognition. Psychophysiology 26:251-269.

Coles MGH, Smid HGOM, Scheffers MK, Otten LJ (1995) Mental chronometry and the study of human information processing. In: Electrophysiology of mind (Rugg MD, Coles MGH, eds), pp 86-131. New York: Oxford UP.

Crone EA, Wendelken C, Donohue SE, Bunge SA (2006a) Neural evidence for dissociable components of task-switching. Cereb Cortex 16:475-486.

Crone EA, Donohue SE, Honomichl R, Wendelken C, Bunge SA (2006b) Brain regions mediating flexible rule use during development. J Neurosci 26:11239-11247.

Crone EA, Wendelken C, Donohue S, van Leijenhorst L, Bunge SA (2006c) Neurocognitive development of the ability to manipulate information in working memory. Proc Natl Acad Sci USA 103:9315-9320.

Deiber MP, Passingham RE, Colebatch JG, Friston KJ, Nixon PD, Frackowiak RS (1991) Cortical areas and the selection of movement: a study with positron emission tomography. Exp Brain Res 84:393-402.

Dosenbach NU, Visscher KM, Palmer ED, Miezin FM, Wenger KK, Kang HC, Burgund ED, Grimes AL, Schlaggar BL, Petersen SE (2006) A core system for the implementation of task sets. Neuron 50:799-812.

Dux PE, Ivanoff J, Asplund CL, Marois R (2006) Isolation of a central bottleneck of information processing with time-resolved FMRI. Neuron 52:1109-1120.

Eriksen BA, Eriksen CW (1974) Effects of noise letters upon the identification of a target letter in nonsearch task. Percept Psychophys 16:143-149.

Forstmann B, Brass M, Koch I, von Cramon DY (2005) Internally generated and directly cued task sets: an investigation with fMRI. Neuropsychologia 43:943-952.

Frith CD, Friston KJ, Liddle PF, Frackowiak RS (1991) Willed action and the prefrontal cortex in man: a study with PET. Proc R Soc Lond B Biol Sci 244:241-246.

Fuggetta G, Pavone E, Walsh V, Kiss M, Eimer M (2006) Cortico-cortical interactions in spatial attention: a combined ERP/TMS study. J Neurophysiol 95:3277-3280.

Fuji N, Mushiake H, Tanji J (2001) Distribution of eye- and arm-movement related neuronal activity in the SEF and in the SMA and the pre-SMA of monkeys. J Neurophysiol 87:2158-2166.

Gratton G, Coles MG, Sirevaag EJ, Eriksen CW, Donchin E (1988) Pre- and poststimulus activation of response channels: a psychophysiological analysis. J Exp Psychol Hum Percept Perform 14:331-344.

Hadland KA, Rushworth MFS, Passingham RE, Jahanshahi M, Rothwell JC (2001) Interference with performance of a response selection task that has no working memory component: an rTMS comparison of the dorsolateral prefrontal and medial frontal cortex. J Cogn Neurosci 13:1097-1108.

He S-Q, Dum RP, Strick PL (1995) Topographic organization of corticospinal projections from the frontal lobe: motor areas on the medial surface of the hemisphere. J Neurosci 15:3284-3306.

Husain M, Parton A, Hodgson TL, Mort D, Rees G (2003) Self-control during response conflict by human supplementary eye field. Nat Neurosci 6:117-118.

Isoda M, Hikosaka O (2007) Switching from automatic to controlled action by monkey medial frontal cortex. Nat Neurosci 10:240-248.

Jahanshahi M, Ridding M, Limousin P, Profice P, Fogel W, Dressler D, Fuller R, Brown RG, Brown P, Rothwell JC (1997) Rapid rate transcranial 
magnetic stimulation-a safety study. Electroencephalogr Clin Neurophysiol 105:422-429.

Kennerley SW, Sakai K, Rushworth MF (2004) Organization of action sequences and the role of the pre-SMA. J Neurophysiol 91:978-993.

Koch G, Franca M, Del Olmo MF, Cheeran B, Milton R, Alvarez Sauco M, Rothwell JC (2006) Time course of functional connectivity between dorsal premotor and contralateral motor cortex during movement selection. J Neurosci 26:7452-7459.

Kornhuber HH, Deecke L (1965) Changes in the brain potential in voluntary movements and passive movements in man: readiness potential and reafferent potentials. Pflügers Arch Gesamte Physiol Menschen Tiere 284:1-17.

Lau HC, Rogers RD, Haggard P, Passingham RE (2004) Attention to intention. Science 303:1208-1210

Liston C, Matalon S, Hare TA, Davidson MC, Casey BJ (2006) Anterior cingulate and posterior parietal cortices are sensitive to dissociable forms of conflict in a task-switching paradigm. Neuron 50:643-653.

Luppino G, Matelli M, Camarda R, Rizzolatti G (1993) Corticospinal connections of area F3 (SMA-proper) and area F6 (pre-SMA) in the macaque monkey. J Comp Neurol 338:114-140.

Marzi CA, Miniussi C, Maravita A, Bertolasi L, Zanette G, Rothwell JC, Sanes JN (1998) Transcranial magnetic stimulation selectively impairs interhemispheric transfer of visuo-motor information in humans. Exp Brain Res 118:435-438.

Miyachi S, Lu X, Inoue S, Iwasaki T, Koike S, Nambu A, Takada M (2005) Organization of multisynaptic inputs from prefrontal cortex to primary motor cortex as revealed by retrograde transneuronal transport of rabies virus. J Neurosci 25:2547-2556.

Nachev P, Rees G, Parton A, Kennard C, Husain M (2005) Volition and conflict in human medial frontal cortex. Curr Biol 15:122-128.

Nakamura K, Sakai K, Hikosaka O (1998) Neuronal activity in the medial frontal cortex during learning of sequential procedures. J Neurophysiol 80:2671-2687.

Requin J, Riehle A, Seal J (1988) Neuronal activity and information processing in motor control: from stages to continuous flow. Biol Psychol 26:179-198.
Rushworth MFS, Paus T, Sipila PK (2001) Attention systems and the organization of the human parietal cortex. J Neurosci 21:5262-5271.

Rushworth MFS, Hadland KA, Paus T, Sipila PK (2002) Role of the human medial frontal cortex in task switching: a combined fMRI and TMS study. J Neurophysiol 87:2577-2592.

Rushworth MFS, Walton ME, Kennerley SW, Bannerman DM (2004) Action sets and decisions in the medial frontal cortex. Trends Cogn Sci 8:410-417.

Sakai K, Passingham RE (2003) Prefrontal interactions reflect future task operations. Nat Neurosci 6:75-81.

Sakai K, Rowe JB, Passingham RE (2002) Active maintenance in prefrontal area 46 creates distractor-resistant memory. Nat Neurosci 5:479-484.

Shima K, Mushiake H, Saito N, Tanji J (1996) Role for cells in the presupplementary motor area in updating motor plans. Proc Natl Acad Sci USA 93:8694-8698.

Silvanto J, Lavie N, Walsh V (2006) Stimulation of the human frontal eye fields modulates sensitivity of extrastriate visual cortex. J Neurophysiol 96:941-945.

Stuphorn V, Schall JD (2006) Executive control of countermanding saccades by the supplementary eye field. Nat Neurosci 9:925-931.

Taylor PC, Nobre AC, Rushworth MF (2007) FEF TMS affects visual cortical activity. Cereb Cortex 17:391-399.

Thaler D, Chen Y, Nixon PD, Stern C, Passingham RE (1995) The functions of the medial premotor cortex. I. Simple learned movements. Exp Brain Res 102:445-460.

Walsh V, Cowey A (2000) Transcranial magnetic stimulation and cognitive neuroscience. Nat Rev Neurosci 1:73-77.

Walsh V, Rushworth MFS (1999) The use of transcranial magnetic stimulation in neuropsychological testing. Neuropsychologia 37:125-135.

Wassermann EM (1998) Risk and safety of repetitive transcranial magnetic stimulation: report and suggested guidelines from the international workshop on the safety of repetitive transcranial magnetic stimulation, June 5-7, 1996. Electroencephalogr Clin Neurophysiol 198:1-16.

Werhahn KJ, Taylor J, Ridding M, Meyer BU, Rothwell JC (1996) Effect of transcranial magnetic stimulation over the cerebellum on the excitability of human motor cortex. Electroencephalogr Clin Neurophysiol 101:58-66. 